\title{
Lung ultrasound and B-lines quantification inaccuracy: $B$ sure to have the right solution
}

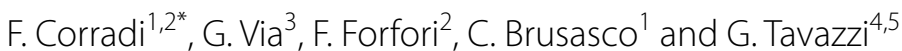

(0) 2020 Springer-Verlag GmbH Germany, part of Springer Nature

\section{Dear Editor,}

We read with great interest the letter form Haaksma and co-workers titled "Lung ultrasound and B-lines: B careful!" [1]. The authors found that lung ultrasound (LUS) reproducibility of B-lines detection with different transducers and raters was poor to moderate and raised a relevant issue.

B-lines are dynamic LUS artifacts, moving and potentially changing appearance over the respiratory cycle, associated with increased extravascular lung water or partial lung loss of aeration. Although the recognition of B-lines and discrimination of A- from B-pattern are simple tasks, the quantification of $\mathrm{B}$-lines and the assessment of their spacing can be challenging: easily counted when few, it becomes impossible to enumerate them precisely when numerous and tending to merge (as often happens in the interstitial-alveolar syndromes).

Semiquantitative methods have been proposed to quantify B-lines based on visual estimation of screen percentage occupied by them [2] or on the presence/absence of their coalescence [3]. These methods may be prone to errors either due to inter-operator "eyeballing" variability or to assessment of their coalescence without considering its overall pleural extension.

The current recommendation to assess the percentage of pleural line occupied by B-lines (rather than counting their maximum number over each ultrasound scan) [2] may also lead to inaccurate results.

At present, no tool is available to quantify the percentage of pleural line occupied by B-lines. This may not be a simple cognitive process because: (1) the distance

\footnotetext{
*Correspondence: francesco.corradi@unipi.it

2 Department of Surgical, Medical and Molecular Pathology and Critical Care Medicine, University of Pisa, Pisa, Italy
}

Full author information is available at the end of the article between two B-lines may not be reliably assessed visually and may change over the respiratory cycle; (2) "coalescence" between two B-lines may be an arguable concept, considering that the same artifact could be interpreted as two close B-lines or a wide B-line; (3) rating all coalescences with the same score, regardless of the percentage of pleura involved, may lead to overestimation of the pathology when this is focal and not ubiquitous, as in ARDS.

The lack of a reference method to objectively quantify B-lines may thus have affected the interpretation of the supposed differences in visualizating them with different probes reported by Haaksma et al.

We believe that the absence of a quantitative scoring system may be overcome by computer-aided measurements of the percentage of pleural line presenting B-line artifacts. This has already been shown to provide a promising and reliable operator-independent assessment of lung surface density (Fig. 1), which seems to outperform previously described subjective scores [4-6].

The next mandatory step will be the automation of this technique, by developing a computer-based clinically easy-to-use tool able to grant an objective pleural line artifacts evaluation. This would reduce inter- and intraobserver variability and create a unique quantification system in order to standardize diagnostic and monitoring scores. Such methodology, supported by artificialintelligence software, has been successfully tested for other ultrasound automated techniques [7]. The potential advantages in terms of faster data collection without increased costs and patients risks are intuitive. The clinical usefulness and importance for lung disease diagnosis and monitoring, in an era fraught with the challenge of pandemic infectious interstitial diseases (such as COVID-19), are easy to guess. 


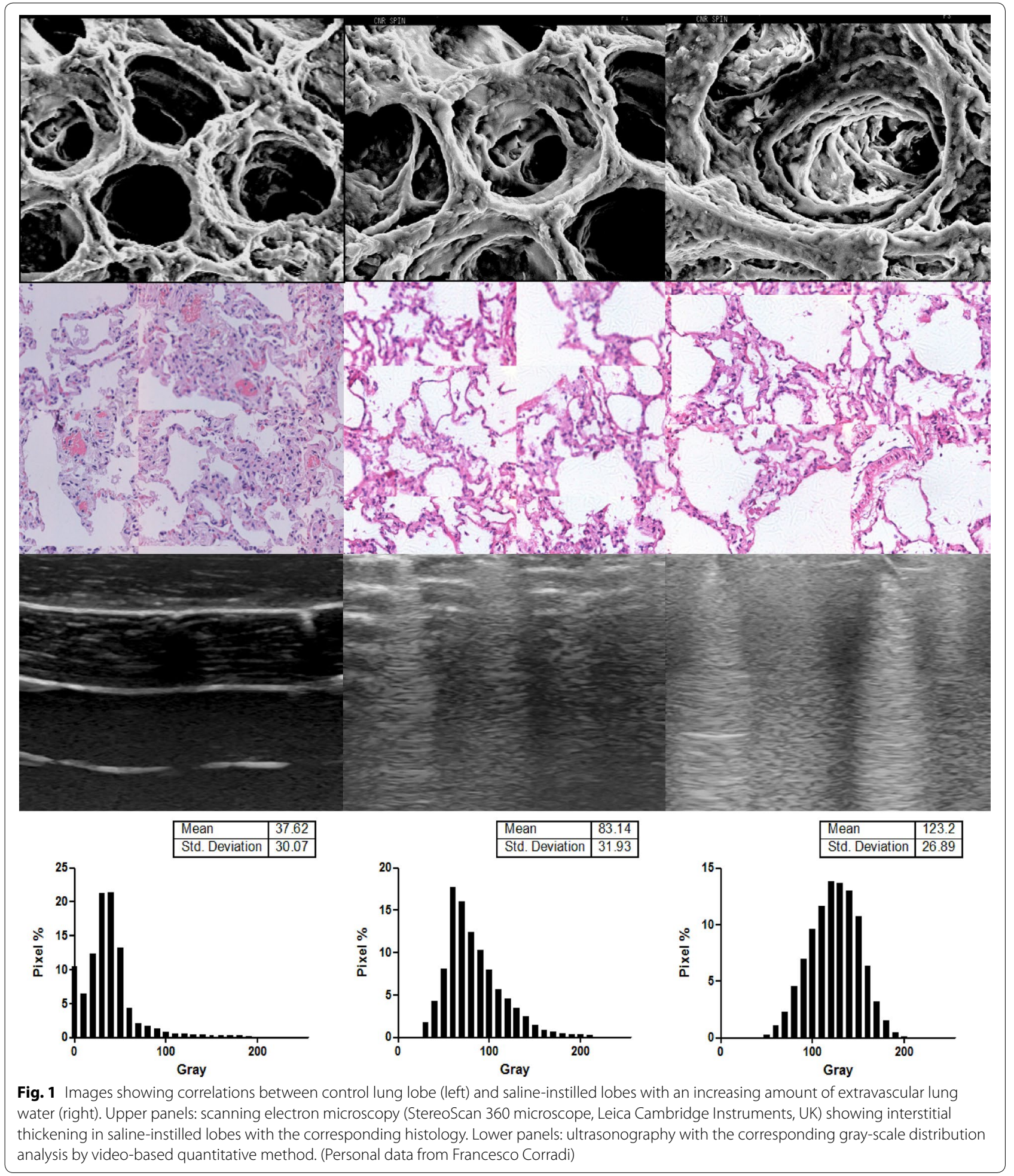




\section{Author details}

${ }^{1}$ Anaesthesia and Intensive Care Unit, Ente Ospedaliero Ospedali Galliera, Genoa, Italy. ${ }^{2}$ Department of Surgical, Medical and Molecular Pathology and Critical Care Medicine, University of Pisa, Pisa, Italy. ${ }^{3}$ Cardiac Anesthesia and Intensive Care, Fondazione Cardiocentro Ticino, Lugano, Switzerland. ${ }^{4}$ Department of Clinical, Surgical, Diagnostic and Pediatric Sciences, University of Pavia, Pavia, Italy. ${ }^{5}$ Anaesthesia, Intensive Care and Pain Therapy, Fondazione IRCCS Policllinico San Matteo, Pavia, Italy.

\section{Compliance with ethical standards}

\section{Conflicts of interest}

All authors declare that they have no conflict of interest.

\section{Publisher's Note}

Springer Nature remains neutral with regard to jurisdictional claims in published maps and institutional affiliations.

\section{Accepted: 7 March 2020}

Published online: 18 March 2020

\section{References}

1. Haaksma ME, Smit JM, Heldeweg MLA, Pisani L, Elbers P, Tuinman PR (2020) Lung ultrasound and B-lines: B careful!. Intensive Care Med 46:544-545. https://doi.org/10.1007/s00134-019-05911-8
2. Gargani L (2011) Lung ultrasound: a new tool for the cardiologist. Cardiovasc Ultrasound 9:6

3. Soummer A, Perbet S, Brisson H, Arbelot C, Constantin JM, Lu Q, Rouby JJ, Lung Ultrasound Study G (2012) Ultrasound assessment of lung aeration loss during a successful weaning trial predicts postextubation distress*. Crit Care Med 40:2064-2072

4. Corradi F, Brusasco C, Garlaschi A, Paparo F, Ball L, Santori G, Pelosi P, Altomonte F, Vezzani A, Brusasco V (2015) Quantitative analysis of lung ultrasonography for the detection of community-acquired pneumonia: a pilot study. Biomed Res Int 2015:868707

5. Corradi F, Brusasco C, Vezzani A, Santori G, Manca T, Ball L, Nicolini F, Gherli T, Brusasco V (2016) Computer-aided quantitative ultrasonography for detection of pulmonary edema in mechanically ventilated cardiac surgery patients. Chest 150:640-651

6. Brusasco C, Santori G, Bruzzo E, Tro R, Robba C, Tavazzi G, Guarracino F, Forfori F, Boccacci P, Corradi F (2019) Quantitative lung ultrasonography: a putative new algorithm for automatic detection and quantification of B-lines. Crit Care 23:288

7. Zhang J, Gajjala S, Agrawal P, Tison GH, Hallock LA, Beussink-Nelson L, Lassen MH, Fan E, Aras MA, Jordan C, Fleischmann KE, Melisko M, Qasim A, Shah SJ, Bajcsy R, Deo RC (2018) Fully automated echocardiogram interpretation in clinical practice. Circulation 138:1623-1635 